\title{
A Co-operative Numerical Analysis of Cultures Considered to Belong to the 'rhodochrous' Taxon
}

\author{
By M. GOODFELLOW, ${ }^{1}$ A. LIND, ${ }^{2}$ HALINA MORDARSKA, ${ }^{3}$ \\ S. PATTYN, ${ }^{4}$ M. TSUKAMURA ${ }^{5}$
}

(Received 22 May 1974; revised I7 July 1974)

\begin{abstract}
SUMMARY
A co-operative taxonomic study has been performed on cultures belonging to the 'rhodochrous complex'. Phenetic data on 98 cultures $(78$ rhodochrous cultures, I 2 marker cultures with the genus designation Mycobacterium and 8 with the designation Nocardia) studied in four laboratories were collected and analysed by numerical taxonomic methods. The precipitinogenic properties and the presence of different types of mycolic acids were analysed independently to establish correlation with the numerical classification. The chemotaxonomic and serological data correlated well with the numerical analyses. The rhodochrous taxon can be distinguished from the genera Mycobacterium and Nocardia, and from the proposed genus Actinomadura; furthermore, it can be divided into at least three homogeneous subgroups. Further studies however, are needed before the question of the generic location of the rhodochrous taxon can be settled and before the taxonomic status of the rhodochrous subclusters can be resolved.
\end{abstract}

\section{INTRODUCTION}

The International Working Group on Mycobacterial Taxonomy (IWGMT) has carried out a number of co-operative taxonomic studies in an attempt to improve the classification and identification of species within the genus Mycobacterium (Wayne et al. I97I; Kubica et al. 1972; Meissner et al. 1974). At an early stage it was recognized that not only species which indisputably belonged to the genus Mycobacterium had to be considered but also taxa with no settled generic niche such as the rhodochrous taxon or 'rhodochrous complex'. The organisms in this group have been classified in the genus Mycobacterium (Gordon \& Mihm, 1957) and in the genus Nocardia (Lechevalier, Horan \& Lechevalier, 1971), while Gordon \& Mihm (I959) characterized the taxon as a 'species in search of a genus'. By 1969 no definitive numerical taxonomic study had been reported on the rhodochrous taxon, so the IWGMT decided to organize a co-operative numerical analysis of cultures considered to belong to the 'rhodochrous complex'. This paper gives the results obtained in a study in which workers from five different laboratories (coded A to E) participated. This investigation was performed using the same 'permissive' philosophy (Wayne et al. 197I) adopted in the earlier studies.

\footnotetext{
1 Department of Microbiology, University of Newcastle upon Tyne, Newcastle upon Tyne NEI 7 RU.

2 Institute of Medical Microbiology, University of Göteborg, Sweden.

3 Ludwik Hirszfeld's Institute of Immunology and Experimental Therapy, Wrocław, Poland.

4 Instituut voor Tropische Geneeskunde, Antwerp, Belgium.

5 The National Sanatorium, Chubu Chest Hospital, Obu, Aichi-ken 474, Japan.
} 
Table I. Species designations and sources of marker cultures

\begin{tabular}{cl}
$\begin{array}{c}\text { ATCC no. } \\
\text { of source }\end{array}$ & \multicolumn{1}{c}{ Designation } \\
6841 & Mycobacterium fortuitum \\
II758 & Mycobacterium phlei \\
14468 & Mycobacterium smegmatis \\
14474 & Mycobacterium flavescens \\
15483 & Mycobacterium vaccae \\
19235 & Mycobacterium borstelense \\
19340 & Mycobacterium diernhoferi \\
19527 & Mycobacterium thermoresistible \\
19686 & Mycobacterium parafortuitum \\
23023 & Mycobacterium peregrinum
\end{tabular}

\author{
ATCC no. \\ of source \\ Designation \\ 23366 Mycobacterium aurum \\ 25969 Mycobacterium album (from R. E. Gordon) \\ I4629 Nocardia caviae \\ 14816 Nocardia pelletieri \\ 19247 Nocardia asteroides \\ 19296 Nocardia brasiliensis \\ 19425 Nocardia madurae \\ 232 I9 Nocardia dassonvillei \\ 25970 Nocardia farcinica (from R. E. Gordon) \\ 2597 I Nocardia farcinica (from R. E. Gordon)
}

\section{METHODS}

Bacterial cultures. One hundred cultures representing the 'rhodochrous complex' and allied taxa were selected from three collections, the American Type Culture Collection, ATCC (32 cultures), the U.S. Department of Health Education, and Welfare Center for Disease Control, C.D.C. (28 cultures), and the collection of Dr R. E. Gordon, R.E.G. (40 cultures). All cultures were lyophilized, coded and distributed by the ATCC as before (Wayne et al. I97I).

Eighty of the test cultures were assigned to the 'rhodochrous complex' (Table $2 a, b, c$ below) and of the 20 marker strains 12 had been classified in the genus Mycobacterium; most of them were rapidly growing mycobacteria belonging to Runyon's group IV (Kubica et al. 1972) and eight were in the genus Nocardia (Table I). Where possible the marker strains were type cultures. Out of the 80 rhodochrous cultures, 28 were designated as 'rhodochrouslike strains', two as 'unidentified strains', whereas the remaining 50 cultures carried 30 different species epithets. Six of the eighty rhodochrous cultures were included as duplicates or triplicates (Table $2 a, b$ ).

Collection and coding of data. Participants studied the cultures using tests of their own choosing. As before (Wayne et al. 197I), participants agreed not to withdraw their data regardless of the final result and agreed that no details of the techniques used would be presented at this stage. The individual batches of data were sent to the co-ordinators who recorded the relevant information into binary form for the numerical analyses. Four laboratories submitted a total of 272 characters per culture but this data included both 'irrelevant' and 'repetitious' characters. Tests in which all of the cultures gave the same result, that is either all positive or all negative responses, were deleted as irrelevant as they have no differential value. Fifty-six tests were carried out by more than one investigator and as they gave substantially similar results only those from a single laboratory were retained. Repetitious data must be eliminated in order to avoid undue weighting of characters. The final $n \times t$ matrix contained 180 characters and 98 operational taxonomic units, o.t.u. (i.e. cultures). The findings of the chemical and serological studies were not included in the pooled data but were reserved for comparison with the results of the numerical analyses.

Computer analyses. The data were examined using the matching coefficient, $S_{M}$, which counts both positive and negative similarities. Initially trials were also made with the similarity coefficient, $S_{J}$, which excludes negative matches, but the results obtained were very similar to those obtained with the $S_{M}$ coefficient and therefore revealed no important additional points of interest. Conventional sorted similarity matrices were obtained using both 


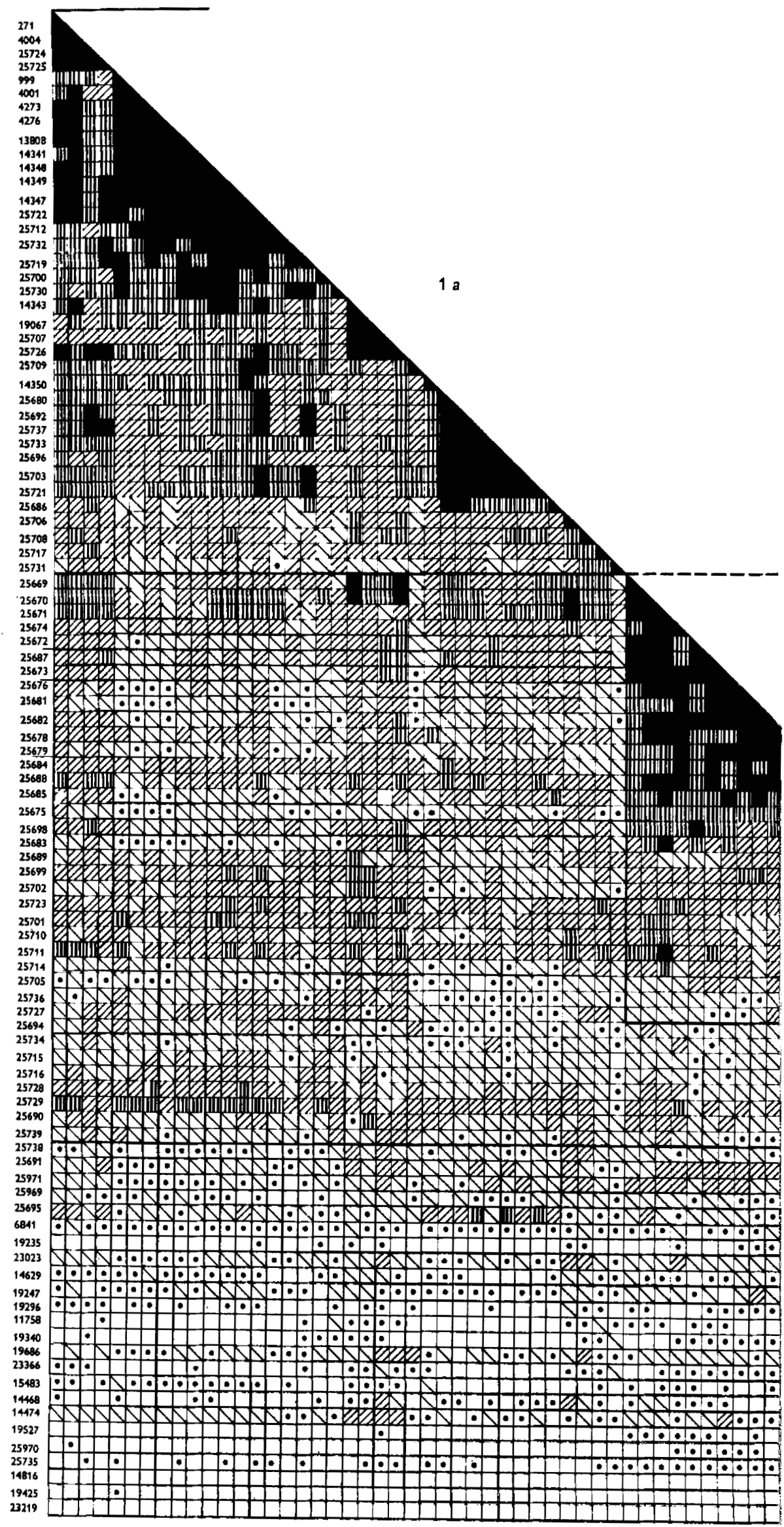

Fig. I. Matching matrix of the 98 te 
$85-100 \%$

गII $80-84 \%$

D $75-79 \%$

D $70-74 \%$

- $65-69 \%$

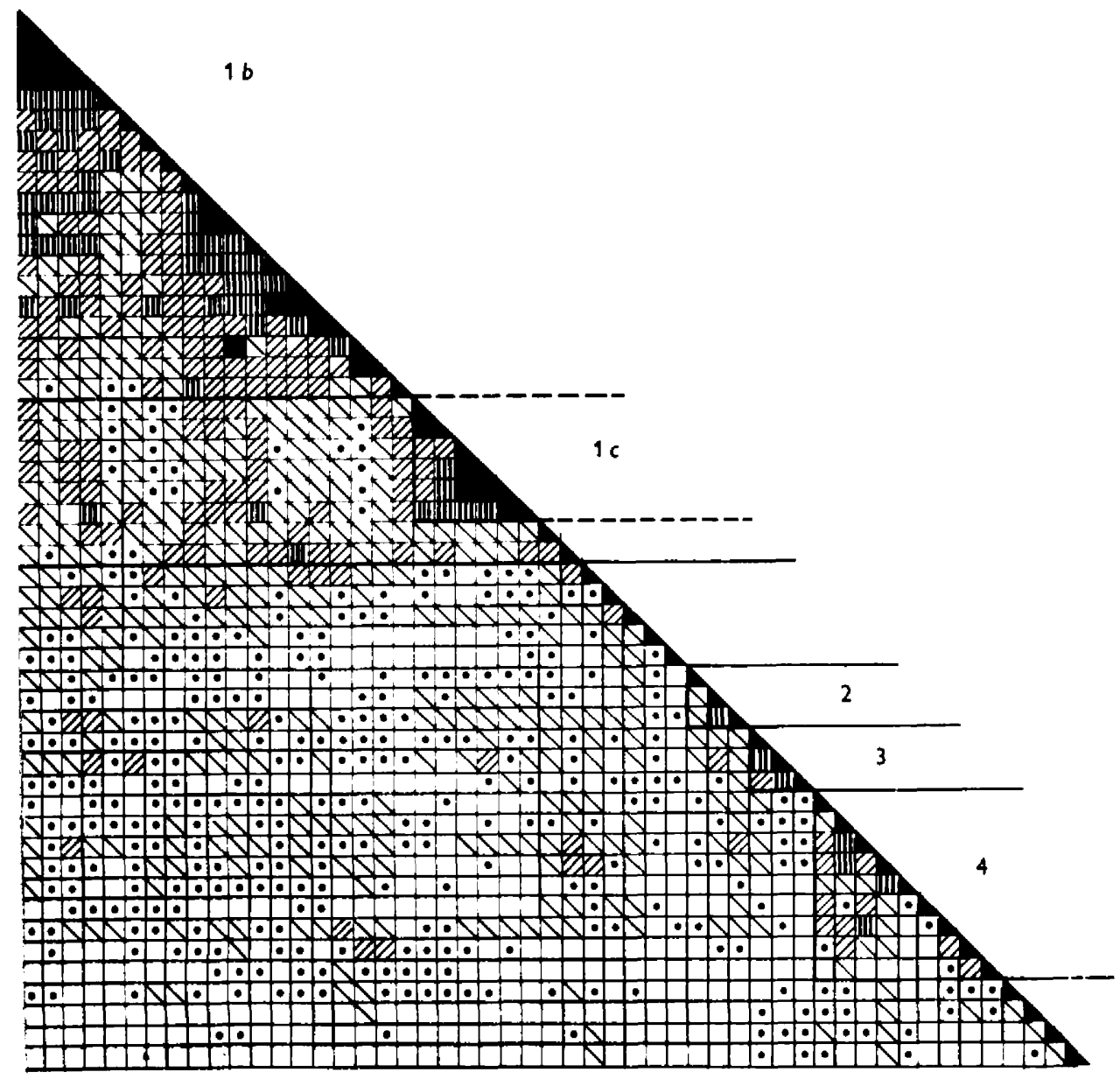

st cultures based on 180 pooled characters. 


single linkage and unweighted average linkage clustering (Sokal \& Sneath, 1963). Since the results of the two clustering techniques were virtually the same only those from the average linkage are considered here.

\section{RESULTS}

\section{Numerical analysis of pooled data from laboratories $A$ to $D$}

Analysis of 180 unit characters and 98 o.t.u. resolved the cultures into four clusters defined at the 75 to $80 \%$ similarity level (S-level) (Fig. I). Most of the cultures in the largest cluster, cluster $\mathrm{I}$, fell into one of three subclusters, I $a, \mathrm{I} b$ or I $c$ (Table $2 a, b, c$ ). The mean intra-group similarity of each cluster and subcluster, and the corresponding inter-group similarities are shown in Table 3 . With the exception of cluster 4 (at $71 \%$ ) mean intra-group similarities were high $(\geqslant 77 \%)$.

Cluster I contains 74 of the 79 cultures provisionally assigned to the rhodochrous complex'. Thirty-seven of these cultures fell into subcluster I $a$ (Table $2 a$ ), 29 into I $b$ (Table $2 b$ ), 6 into I $c$ (Table $2 c$ ), while 2 cultures possibly formed the nucleus of a fourth subcluster (Table $2 c$ ). None of the marker strains were recovered in this cluster. The mean intra-group similarities for the three subgroups in cluster I are 8I, 79 and $83 \%$ respectively (Table 3), clearly separating this cluster from clusters 2,3 and $4(\leqslant 70 \%)$.

Cluster 2 contains three cultures belonging to Runyon's group IV, namely Mycobacterium borstelense (ATCCI9235), M. fortuitum (ATCC684I) and $M$. peregrinum (ATCC23023).

Cluster 3 consists of three nocardiae cultures, Nocardia asteroides (ATCCI9247), $N$. brasiliensis (ATCCI9296) and N. caviae (ATCCI4629).

Cluster 4 includes nine cultures. Eight are rapidly growing mycobacteria: Mycobacterium aurum (ATCC23366), $M$. diernhoferi (ATCC19340), M. flavescens (ATCCI4474), M. parafortuitum (ATCCI9686), M. phlei (ATCCI I758), M. smegmatis (ATCCI4468), $M$. thermoresistibile (ATCCI9527), and $M$. vaccae (ATCCI 5483); the ninth is one of the two Nocardia farcinica cultures (ATCC25970).

Nine cultures were not recovered in the defined clusters, namely Corynebacterium fascians (ATCC25738), Mycobacterium album (ATCC25969), Nocardia dassonvillei (ATCC23219), N. farcinica (ATCC2597I), N. madurae (ATCC19425), N. pelletieri (ATCC14816), N. rhodnii (ATCC25735), and two 'rhodochrous-like strains' (ATCC2569I and ATCC25695).

Numerical analysis of data from laboratory $A$. In this laboratory the cultures were examined for I04 characters, 12 of which were deleted as irrelevant. Nintey-three of the 98 cultures studied fell into five clusters defined at the 75 to $80 \% \mathrm{~S}$-level. The largest cluster was sharply separated from the other clusters and contained 77 cultures initially assigned to the 'rhodochrous complex'. Most of the rhodochrous cultures were recovered in three homogeneous subclusters which were almost identical in composition to those obtained from the analysis of the pooled data. A fourth but diffuse subcluster was formed by eight cultures, namely C. fascians (ATCC25738 and ATCC25739), Nocardia sp. (ATCC25707), M. album (ATCC25969), Proactinomyces spp. (ATCC25708, ATCC25709 and ATCC257I0) and a 'rhodochrous-like strain' (ATCC25683). All the duplicate and triplicate cultures were found in the relevant subcluster.

Most of the marker strains were recovered in the four remaining clusters. The three Nocardia cultures were again recovered as a distinct cluster, and most of the mycobacteria were once more distributed between two clusters. In this analysis, however, a fifth cluster was formed comprising Mycobacterium vaccae (ATCCI5483) and a 'rhodochrous-like strain' (ATCC25691). The cultures representing Nocardia dassonvillei, N.farcinica (ATCC2597I), $N$. madurae, $N$. pelletieri and $N$. rhodnii once again remained outside the defined clusters. 
Table 2. Cultures assigned to cluster I

(a) Cultures assigned to subcluster I $a$

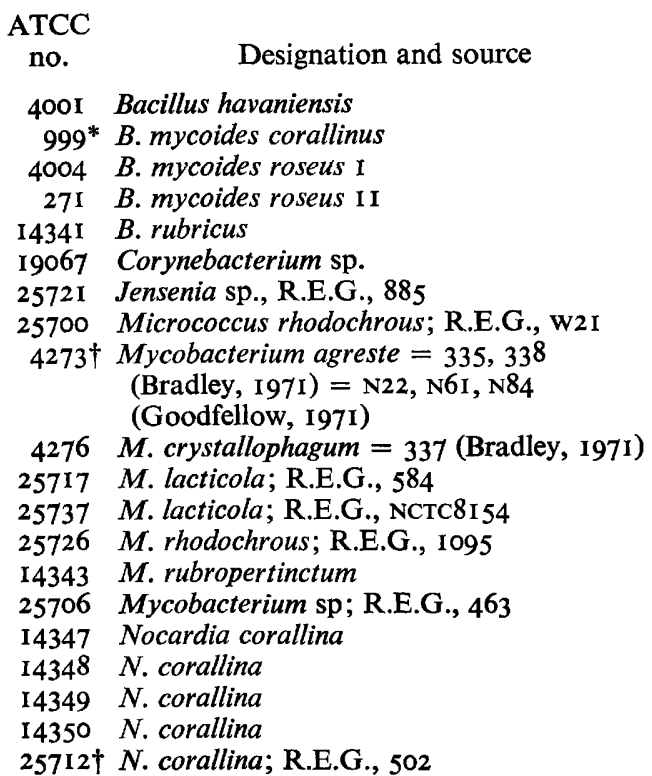

ATCC

no. Designation and source

25719* N. corallina; R.E.G., 624

25730* N. corallina; R.E.G., W3406

$25732 \dagger N$. corallina; R.G.E., W3408

25731 N. erythropolis; R.E.G., W3407 = ATCC4277 $=474($ Bradley, I97I $)=$ N23, NI08 (Goodfellow, I97I)

25703 N. rubra; R.E.G., 415

25733 N. rubra; R.E.G., W3639 = NIIO

(Goodfellow, 197I)

25707 Nocardia sp; R.E.G., 482

25724 Nocardia sp; R.E.G., I082R

25725 Nocardia sp; R.E.G., I082s

25708 Proactinomyces erythropolis; R.E.G., 492

25709 Proactinomyces sp; R.E.G., 493

I3808 Rhodococcus rhodochrous $=$ N54

(Goodfellow, 1971)

25680 'rhodochrous'; C.D.C., T2568-6

25686 'rhodochrous'; C.D.C., Rhod. 2

25692 'rhodochrous'; C.D.C., T227-6

25696 'rhodochrous'; C.D.C., T2059-6

25722 Unidentified strain; R.E.G., 1022

(b) Cultures assigned to subcluster $\mathrm{I} b$

ATCC

no.

25705 Mycobacterium eos; R.E.G., 462

25723 $\$$ M. rhodochrous; R.E.G., 1054

$25702 M$. rubrum; R.E.G., 384

25736 Mycobacterium sp., R.E.G., NCTC8I39

257I4 Nocardia globerula; R.E.G., 544

25710 Proactinomyces globerula; R.E.G., $494=$ NIo9 (Goodfellow, 197I)

257I I P. globerula; R.E.G., 495

25669 'rhodochrous'; C.D.C., T334-5

25670 'rhodochrous'; C.D.C., T454-5

2567I 'rhodochrous'; C.D.C., T456-5

25672 'rhodochrous'; C.D.C., T644-5

25673 'rhodochrous'; C.D.C., T260-6

25674 'rhodochrous'; C.D.C., T262-6

25675 'rhodochrous'; C.D.C., T258-6

25676 'rhodochrous'; C.D.C., T2060-6

$\begin{array}{cc}\begin{array}{c}\text { ATCC } \\ \text { no. }\end{array} & \text { Designation and source } \\ 25678 & \text { 'rhodochrous'; C.D.C., T2338-6 } \\ 25679 & \text { 'rhodochrous'; C.D.C., T2544-6 } \\ 25681 & \text { 'rhodochrous'; C.D.C., T2569-6 } \\ 25682 & \text { 'rhodochrous'; C.D.C., T610-7 } \\ 25683 & \text { 'rhodochrous'; C.D.C., T875-7 } \\ 256847 & \text { 'rhodochrous'; C.D.C., T957-7 } \\ 25685 & \text { 'rhodochrous'; C.D.C., T452-8 } \\ 25687 & \text { 'rhodochrous'; C.D.C., T312-2 } \\ 25688 & \text { 'rhodochrous'; C.D.C., T394-2 } \\ 25689 & \text { 'rhodochrous'; T572-5 } \\ 25698 & \text { 'rhodochrous'; C.D.C., T954-7 } \\ 25699 & \text { 'rhodochrous'; C.D.C., T980-7 } \\ 25701 & \text { Unidentified strain; R.E.G., 382 } \\ 25727 & \text { Corynebacterium rubrum; R.E.G., } \\ & \text { I240 = N59 (Goodfellow, I97I) }\end{array}$

ATCC

no. ce

(c) Cultures assigned to subcluster $\mathrm{I} c$, to cluster I and those not recovered in a defined cluster

ATCC

no.

Designation and source

Subcluster I $c$

$25694 \S$ Corynebacterium equi, C.D.C., T675-6

25729 C. equi, R.E.G., I62I

$25734 \S$ C. equi, R.E.G., NCTC42I9

25715 Nocardia restrictus; R.E.G., 545

25728 N. restrictus; R.E.G., I 256

25716 Proactinomyces restrictus; R.E.G., 566
ATCC

no. Designation and source

Cluster I

25690 'rhodochrous'; C.D.C., T595-5

25739 Corynebacterium fascians; R.E.G., 1300

Not clustered

2569I 'rhodochrous'; C.D.C., T204-6

25695 'rhodochrous'; C.D.C., T2054-6

25735 Nocardia rhodnii; R.E.G., NCTC6I 17

25738 Corynebacterium fascians; R.E.G., I 2974

ATCCI 84 Micrococcus rhodochrous and ATCC25704 Nocardia globerula (R.E.G., 417) were excluded as insufficient data was received on them.

$*, \dagger$ Triplicate cultures.

$\ddagger$ \& Duplicate cultures. 
Table 3. Similarity levels $(\%$ S-level $)$

These are expressed as mean inter- and intra-group similarities, with standard deviations, for the six clusters and subclusters. The S-levels are rounded to the nearest I \%.

$\begin{array}{cc}\text { Cluster I } a & 8 \mathrm{I} \pm 4 \cdot 8 \\ \mathrm{I} b & 75 \pm 4 \cdot \mathrm{I} \\ \mathrm{I} c & 74 \pm 3 \cdot 9 \\ 2 & 66 \pm 4 \cdot 2 \\ 3 & 67 \pm 3 \cdot 3 \\ 4 & 65 \pm 5 \cdot \mathrm{I} \\ & \mathrm{I} a\end{array}$

$$
\begin{aligned}
& 79 \pm 5 \cdot 4 \\
& 74 \pm 3 \cdot 3 \\
& 68 \pm 4 \cdot 1 \\
& 68 \pm 3 \cdot 9 \\
& 67 \pm 3 \cdot 7
\end{aligned}
$$
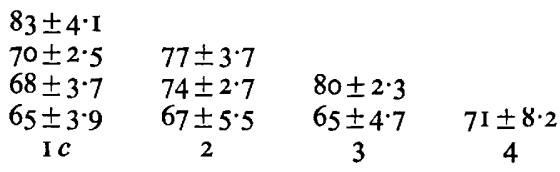

Numerical analysis of data from laboratory $B$. In this laboratory the cultures were examined for 108 characters, but for computation 20 of these were deleted because they gave results which were either $100 \%$ positive or $100 \%$ negative. At the 75 to $80 \%$ S-level five clusters were defined. The rhodochrous cultures were again found in three subclusters within a cluster sharply separated from the remaining clusters. Cluster 2 was recovered as in the analysis of the pooled data but also contained two additional marker cultures, $M$. parafortuitum and $N$. farcinica (ATCC2579I). The three Nocardia cultures were again found in cluster 3 but the second Mycobacterium cluster was smaller than before and only contained four cultures, $M$. aurum, M. diernhoferi, $M$. phlei and $M$. vaccae. The fifth cluster was heterogeneous and contained the slower-growing cultures $M$. flavescens, $M$. thermoresistibile, $N$. pelletieri and the rhodochrous culture ATCC25705. In this analysis the marker strains $N$. dassonvillei, N. farcinica (ATCC25970), N. madurae and M. smegmatis were not grouped in any of the defined clusters.

Numerical analysis of data from laboratory $C$. In this laboratory the test cultures were examined for 28 unit characters including four serological ones. Three clusters were recovered at the 75 to $80 \% \mathrm{~S}$-level. Once more all the rhodochrous cultures were found in a distinct cluster, but in this analysis the four marker cultures $M$. abscessus, $M$. diernhoferi, $M$. flavescens and $M$. parafortuitum were also included in cluster I. Subclusters I $a$ and I $b$ were again distinguishable but the final subcluster $\mathrm{r} c$ was now found as a recognizable entity in subcluster $\mathrm{I} a$. With a single exception, the duplicate and triplicate cultures were found in the relevant subcluster.

Cluster 3 was again recovered, but in this analysis also contained the marker strains $N$. dassonvillei, $N$. madurae, and $N$. pelletieri. With a single exception, the Mycobacterium marker cultures and $N$. farcinica (ATCC2597I) were grouped together in a large heterogeneous cluster. The remaining culture, M. thermoresistible, did not fall into any of the defined clusters.

Numerical analysis of data from laboratory $D$. The data from this laboratory were used to form an $n \times t$ matrix consisting of 98 cultures and 32 unit characters. The results were very similar to those from laboratory $\mathrm{C}$. The rhodochrous cultures again fell into a distinct cluster and the subclusters were again recognizable. The Nocardia and Mycobacterium marker cultures were separate from one another and from the rhodochrous cultures.

\section{Comparative analyses of independent data}

Serological studies performed by laboratory $C$. This laboratory also analysed the cultures serologically by means of a comparative immuno-diffusion method using 12 serological reference systems representing Mycobacterium bovis var. BCG, M. microti, M. kansasii, M. marianum, M. avium, $M$. fortuitum, $M$. phlei, $N$. asteroides, $N$. caviae, and three members 
Table 4. Number of cultures showing cross-reacting precipitinogens to mycobacterial, ${ }^{*}$ rhodochrous and nocardia cultures used as references for comparative immunodiffusion analyses

No. of precipitates of the reference system showing deviation phenomena are given in parentheses.

Species

\begin{tabular}{|c|c|c|c|c|c|c|c|c|c|c|c|}
\hline \multirow[b]{2}{*}{ Cluster } & \multirow[b]{2}{*}{$\begin{array}{l}\text { No. of } \\
\text { cultures }\end{array}$} & \multirow{2}{*}{$\begin{array}{c}M . \\
\text { bovis } \\
\text { var. } \\
B C G \\
\text { (I) }\end{array}$} & \multirow{2}{*}{$\begin{array}{l}M . \\
\text { microti } \\
\text { (I) }\end{array}$} & \multirow{2}{*}{$\begin{array}{l}M . \\
\text { mari- } \\
\text { anum } \\
(\mathrm{I})\end{array}$} & \multicolumn{2}{|c|}{$\overbrace{}^{M . \text { avium }}$} & \multicolumn{4}{|c|}{ M. fortuitum } & \multirow{2}{*}{$\begin{array}{c}\text { M. phlei } \\
\text { (I) }\end{array}$} \\
\hline & & & & & (I) & $(2)$ & (I) & (2) & (3) & (5) & \\
\hline I $a$ & 37 & 2 & 一 & 一 & 一 & - & I & - & 一 & - & - \\
\hline I $b$ & 29 & 3 & - & 一 & - & - & 一 & - & 一 & 一 & 一 \\
\hline I $c$ & 6 & $\mathbf{I}$ & - & - & - & 一 & 一 & - & 一 & 一 & 一 \\
\hline $\mathbf{I} \dagger$ & 2 & - & 一 & I & 一 & 一 & - & - & - & - & 一 \\
\hline 2 & 3 & I & - & 一 & $\longrightarrow$ & 3 & $\mathbf{I}$ & 一 & 一 & $\mathbf{I}$ & - \\
\hline 3 & 3 & - & - & I & I & I & 一 & - & - & - & - \\
\hline 4 & 9 & 3 & I & 2 & 3 & $\mathbf{I}$ & - & 3 & 一 & - & I \\
\hline Unclustered & 9 & I & - & I & 二 & I & 一 & - & I & 一 & - \\
\hline
\end{tabular}

' $M$.' rhodochrous

' $N$.'pellegrino ' $N$.' polychromogenes

$\begin{array}{rrrrrrrrrrrrrr}(\mathrm{I}) & (2) & (3) & (4) & (5) & \text { (I) } & (2) & \text { (3) } & \text { (4) } & \text { (I) } & \text { (2) } & \text { (3) } & \text { (4) } & \text { (5) } \\ 24 & 2 & \text { I } & \text { I } & - & \text { II } & 5 & \text { I } & \text { I } & - & - & - & - & - \\ 2 & \text { I6 } & 5 & \text { I } & \text { I } & \text { I } & \text { I } & - & - & - & 2 & 5 & 6 & 6 \\ 5 & - & - & - & - & \text { I } & - & - & - & - & - & - & - & - \\ \text { I } & - & - & - & - & - & - & - & - & - & - & - & - & - \\ - & - & - & - & - & - & - & - & - & - & - & - & - & - \\ - & - & - & - & - & 2 & - & - & - & - & - & - & - & - \\ - & - & - & - & - & - & - & - & - & - & - & - & - & - \\ 4 & - & - & - & - & 3 & - & \text { I } & - & - & - & - & - & -\end{array}$

N. asteroides $\quad$ N. caviae

(1) (2) (3) (1) (6)

$\begin{array}{crrrrrr}\text { I } a & 37 & 8 & \text { I } & - & - & - \\ \text { I } b & 29 & \text { I } 4 & 5 & - & 4 & - \\ \text { I } c & 6 & - & - & - & - & - \\ \text { I } \dagger & 2 & - & - & - & - & - \\ 2 & 3 & - & - & - & - & - \\ 3 & 3 & \text { I } & \text { I } & \text { I } & - & \text { I } \\ 4 & 9 & \text { I } & \text { I } & - & 3 & - \\ \text { Unclustered } & 9 & 2 & - & - & - & -\end{array}$

* The results obtained with the $M$. kansasii reference system are not included since no culture caused deviation phenomena.

$\uparrow$ Belonging to cluster I but not falling into subclusters $\mathrm{I} a$, I $b$ or I $c$.

of the 'rhodochrous complex', ' $M$.' rhodochrous, ' $N$.' pellegrino and ' $N$.' polychromogenes (Lind, I96I; Norlin, Lind \& Ouchterlony, 1969; Ridell, 1974). In these tests the number of precipitates of the reference system which showed deviation due to the influence of a corresponding precipitinogen in the culture under study were counted. Thus, the total number of precipitates between the well with the antigen under test and the antiserum well was not taken into consideration.

The results of the serological studies were compared with those obtained from the computer analysis of the pooled data (Table 4). Of the 74 cultures belonging to cluster $\mathrm{I}$, one 
had a precipitinogen in common with $M$. marianum, six had one precipitinogen in common with $M$. bovis var. $B C G$, and one of the latter also shared a precipitinogen in common with $M$. fortuitum. None of the remaining 67 cultures had demonstrable precipitinogens in common with the seven mycobacterial reference systems.

Sixty-three of the 74 rhodochrous cultures demonstrated I to 5 precipitinogens in common with one or more of the three 'rhodochrous complex' reference systems. It is noteworthy that while 19 of the cultures belonging to subcluster $\mathrm{I} b$ had influence upon 2 to 5 of the precipitates of the ' $N$.' polychromogenes system none of the 37 subcluster I $a$ cultures showed any reaction. A similar division was obtained with the ' $M$.' rhodochrous reference system, where 23 of the 29 subcluster I $b$ cultures but only four of the subcluster I $a$ cultures influenced two or more precipitates. Similarly, when tested with the Nocardia reference systems the subcluster $\mathrm{I} b$ cultures were more reactive (20 out of 29 ) than those belonging to subcluster I $a$ ( 9 out of 37). The cluster I cultures, therefore, reacted much more frequently in analyses with the Nocardia reference systems ( 29 cultures) than with the mycobacterial reference systems ( 7 cultures).

The cultures in clusters 2,3 and 4 , which contained most of the mycobacterial and nocardia marker strains, did not react with the ' $N$.' polychromogenes or ' $M$.' rhodochrous reference patterns, and in only two instances reacted with the ' $N$.' pellegrino system. The two $N$. farcinica cultures had one demonstrable precipitinogen in common with $M$. bovis var. $B C G$ and two or three in common with $M$. fortuitum, and culture ATCC2597I had two precipitinogens in common with the $M$. avium reference system. Three of the Nocardia cultures, $N$. dassonvillei, $N$. madurae and $N$. pelletieri, had little serological affinity with any of the reference systems, though the latter two cultures had one demonstrable precipitinogen in common with the ' $N$.' pellegrino reference system.

Chemotaxonomic studies performed by laboratory $E$. In this laboratory, whole cell hydrolysates were analysed for the presence of the sugars arabinose and galactose, and for mesoand LL-diaminopimelic acid (DAP). In addition dried cells were examined to see if they contained true mycolic acids (i.e. containing 80 to 90 carbon atoms) or free nocardomycolic acids (40 to 60 carbon atoms). Nocardomycolic acids were detected using a thin-layer chromatographic method which distinguishes between two of the lipid LCN-A (lipid characteristic of Nocardia) analogues, lipid LCN-A type $a$ (asteroides) and lipid LCN-A type $c$ (calcarea) (Mordarska, Mordarksi \& Goodfellow, 1972). LCN-A lipids have properties consistent with those of free nocardomycolic acids (Goodfellow, Minnikin, Patel \& Mordarska, I973).

Correlation of the chemotaxonomic data with the computer-generated clusters and subclusters showed that all cultures in the four clusters contained meso-DAP, arabinose and galactose (Table 5). Lipid LCN-A was detected in all the cultures classified in clusters $\mathrm{I}$ and 3 , and true mycolic acids in all the marker cultures of Mycobacterium. Subclusters I $a$ and I $c$ could, however, be separated from subcluster $\mathrm{r} b$ by the type of lipid LCN-A they contained. Lipid LCN-A type $c$ was found in all the subcluster I $c$ cultures and in 30 out of 37 subcluster I $a$ cultures, whereas lipid LCN-A type $a$ was mainly confined to cultures classified in subgroup I $b$. The analogue with the higher $R_{F}$ value, lipid LCN-A type $a$, was also detected in the Nocardia cultures of cluster 3 . The marker cultures labelled $N$. dassonvillei, $N$. madurae and $N$. pelletieri have a cell wall type I I and do not contain any type of mycolic acid.

Characters with greatest resolving power for separation of the rhodochrous taxon from Mycobacterium and Nocardia. Eighteen characters were chosen as having the greatest resolving power for the separation of cultures assigned to the four clusters (Table 6). Widely 
Table 5. Distribution of cultures by chemotaxonomy (Laboratory E) and phenetic clustering behaviour

\begin{tabular}{|c|c|c|c|c|c|c|}
\hline Cluster & $\begin{array}{l}\text { Total no. } \\
\text { of cultures }\end{array}$ & $\begin{array}{l}\text { No. of } \\
\text { cultures } \\
\text { analysed }\end{array}$ & $\begin{array}{l}\text { Arabinose } \\
\text { galactose } \\
\text { meso-DAP }\end{array}$ & $\begin{array}{c}\text { LCN-A } \\
\text { type } a\end{array}$ & $\begin{array}{c}\text { LCN-A } \\
\text { type } c\end{array}$ & $\begin{array}{l}\text { Mycolic } \\
\text { acids } \\
(\mathrm{C} 80-90)\end{array}$ \\
\hline I $a$ & 37 & 33 & 33 & 3 & 30 & 0 \\
\hline I $b$ & 29 & 26 & 26 & 24 & 2 & 0 \\
\hline I $c$ & 6 & 6 & 6 & 0 & 6 & 0 \\
\hline I* $^{*}$ & 2 & I & I & I & 0 & 0 \\
\hline 2 & 3 & 3 & 3 & 0 & 0 & 3 \\
\hline 3 & 3 & 3 & 3 & 3 & 0 & 0 \\
\hline 4 & 9 & 6 & 6 & 0 & 0 & 6 \\
\hline
\end{tabular}

* Belonging to cluster $\mathrm{I}$ but not falling into subclusters $\mathrm{I} a$, I $b$ or I $c$.

Table 6. Distribution of $\mathrm{I} 8$ characters with the greatest resolving power among the four main clusters

Character

Staining and colony morphology

Acid-fast

Aerial hyphae

Colonies pink, red, orange

Growth

At $10^{\circ} \mathrm{C}$

On ethanol as single carbon source

On sucrose as single carbon source

Lipid analyses

LCN-A, type $a$

Mycolic acids (C80-90)

Enzymic activity

Benzamidase

Urease

Isonicotinamidase

Salicylamidase

Allantoinase

Succinamidase

Malonamidase

$\beta$-Esterase

Growth on $7 \% \mathrm{NaCl}$

Susceptibility to ro i.u. penicillin

\begin{tabular}{|c|c|c|c|}
\hline \multicolumn{4}{|c|}{ No. positive* $(\%)$} \\
\hline $\begin{array}{c}\text { Cluster I } \\
(72)\end{array}$ & $\begin{array}{c}\text { Cluster } 2 \\
\text { (3) }\end{array}$ & $\begin{array}{c}\text { Cluster } 3 \\
\text { (3) }\end{array}$ & $\begin{array}{c}\text { Cluster } \\
\text { (9) }\end{array}$ \\
\hline 0 & 100 & 0 & 100 \\
\hline 0 & 0 & 100 & 0 \\
\hline 94 & 0 & 100 & I I \\
\hline 99 & 0 & 100 & 0 \\
\hline 93 & 0 & 0 & 22 \\
\hline 100 & 33 & 0 & 22 \\
\hline
\end{tabular}

$\begin{array}{crrc}38 \dagger & 0 & 100 & 0 \\ 0 & 100 & 0 & 100+\end{array}$

$\begin{array}{rrrr}2 & 100 & 100 & 44 \\ 93 & 100 & 100 & 78 \\ \text { I } & 67 & 100 & 44 \\ \text { I } & 33 & 100 & 33 \\ 0 & 0 & 100 & 0 \\ \text { I } & 67 & 100 & 44 \\ \text { I } & 67 & 100 & 33 \\ 50 & 100 & 0 & 78 \\ 71 & 0 & 33 & 0 \\ 86 & 0 & 0 & 0\end{array}$

* No. of cultures analysed is given in parentheses.

$\dagger 60$ cultures analysed.

$\ddagger 6$ cultures analysed.

different taxonomic criteria (staining and colony morphology, growth tests, lipid analyses, enzymic resistance tests) all provide information of diagnostic value.

Characters with greatest resolving power for separation of the subclusters within the rhodochrous taxon (cluster I). Fourteen characters were chosen as the most useful for the differentiation of the three subclusters of cluster I (Table 7). Tyrosine hydrolysis and the lipid LCN tests are particularly useful in distinguishing subclusters $\mathrm{x} a$ and $\mathrm{x} b$. 
Table 7. Distribution of 14 characters with the greatest resolving power among the three subgroups of the rhodochrous taxon (cluster I)

Character $\overbrace{\begin{array}{c}\text { Cluster I } a \\ (37)\end{array}}^{\text {No. positive }(\%)}$

Growth on the following as single carbon

source

Maltose

Mannitol

Sorbitol

Trehalose

2,3-Butylene glycol

$m$-Hydroxybenzoic acid

Sebacic acid

Sodium lactate

Testosterone

Growth on the following as single

carbon/nitrogen source

Monoethanolamine

Lipid analyses

LCN-A, type $a$

LCN-A, type $c$

Enzymic activity

Ferric ammonium citrate uptake

Tyrosine hydrolysis

$\begin{array}{rrr}100 & 100 & 0 \\ 100 & 55 & 0 \\ 100 & 55 & 0 \\ 92 & 86 & 0 \\ 100 & 69 & 0 \\ 70 & 10 & 15 \\ 84 & 86 & 0 \\ 89 & 21 & 67 \\ 81 & 7 & 33\end{array}$

$\begin{array}{rrr}43 & 93 & 0 \\ & & \\ 9 \dagger & 92 \ddagger & 0 \\ 91 \dagger & 8 \ddagger & 100 \\ & & \\ 84 & 69 & 0 \\ 89 & 0 & 100\end{array}$

* No. of cultures analysed is given in parentheses.

$\dagger 33$ cultures analysed.

$\div 26$ cultures analysed.

\section{DISCUSSION}

Cultures considered to belong to the 'rhodochrous complex' carry a host of generic and species names and since the late 1950 s have challenged taxonomists interested in the actinomycetes. The problem of a generic niche for rhodochrous cultures has exercised investigators interested in the genus Nocardia as well as those involved with the genus Mycobacterium. Although individual studies carried out in the late 1950 s and 1960s furthered our knowledge of the characteristics of the rhodochrous taxon they did not resolve the taxonomic dilemma (Gordon, 1966; Magnusson, I962; Gordon \& Mihm, 1957, 1959). Eventually, several workers independently recognized that the numerical taxonomic procedure was particularly suited to solving taxonomic problems of this nature and, not surprisingly, the results of a number of numerical taxonomic studies have been published during the course of this co-operative project.

Bradley (I97I) found that cultures referred to the 'rhodochrous complex' were more closely allied with $N$. asteroides and $N$. farcinica than to $M$. tuberculosis. He also concluded that the rhodochrous taxon was heterogeneous and consisted of at least two clusters, one typified by $N$. corallina and $N$. rubra, the other by $N$. erythropolis. The DNA from strains recovered in the first taxon were found to have 66 to $68 \%$ guanine plus cytosine (GC) whereas those in the latter contained $6 \mathrm{I}$ to $63 \% \mathrm{GC}$.

In another numerical taxonomic study, Tsukamura (197I) proposed the genus Gordona for a number of 'rhodochrous-like' organisms. However, only six marker cultures of ' $M$.' rhodochrous were included in this study and none of these clustered with the defined species. 
Representatives of the genus Gordona were not included in the present study and further work is required to determine the relationship of the genus to the rhodochrous taxon. In a more extensive study (Tsukamura, 1973), 20 ' $M$.' rhodochrous cultures were included; Io were classified in a new species Gordona rhodochroa and 2 assigned to a renamed taxon $G$. rubropertincta. The ten strains in the former species were all recovered in subcluster i $a$ and the other two in subcluster $\mathrm{I} b$. Tsukamura did, however, concede that the epithet Gordona for rhodochrous cultures (Goodfellow, Fleming \& Sackin, 1972) might be incorrect.

The most comprehensive numerical taxonomic studies on the 'rhodochrous complex' to date have been presented by Goodfellow (I97I) and Goodfellow et al. (1972). In the earlier study the rhodochrous taxon was clearly delineated from the genera Nocardia, Actinomadura and Oerskovia, and in the later one a demarcation between the rhodochrous taxon and mycobacteria referred to Runyon's group IV was ascertained. Furthermore, the cluster containing the rhodochrous cultures fell into a number of subclusters, three of which were homogeneous. However, Goodfellow et al. (1972) and Cross \& Goodfellow (1973) considered that additional evidence was required before the generic status of the ' $M$ '. rhodochrous taxon could be resolved.

Although markedly different test cultures were used, the results of this co-operative study are in good agreement with earlier findings. In all cases the rhodochrous cluster was defined around the $80 \%$ S-level, and the inter-group similarities between the rhodochrous cluster and clusters containing group IV mycobacteria were between 60 and $65 \%$. Because different rhodochrous cultures were studied it was difficult to compare the subclusters recovered in the various studies. However, subcluster I $a$ (Table 2) can be equated to N. rubra (Bradley, I97I), to subcluster ${ }_{14} \mathrm{C}$ (Goodfellow, 197I) and to G. rhodochroa (Tsukamura, 1973). Similarly, $N$. erythropolis (Bradley, I97I) appears to be the same taxon as subcluster I4D (Goodfellow, I97I). Subcluster I4A (Goodfellow, I97I), which contained cultures received as N. pellegrino, had no counterpart in the co-operative study. Tacquet et al. (I97I) recovered cultures of ' $M$.' pellegrino in one of two subclusters in a rhodochrous cluster clearly differentiated from clusters containing mycobacteria and nocardiae.

The results from the individual laboratories yielded clusters in general agreement with those obtained from the analysis of the pooled data. This was even the case with laboratories $\mathrm{C}$ and D which examined only 28 and 32 unit characters respectively, a number of characters which would normally be considered insufficient for a numerical taxonomic study. Thus, a wealth of numerical taxonomic data has been obtained which shows that the 'rhodochrous complex' forms a taxon which is distinct both from the genus Mycobacterium and from the genus Nocardia, and data from independent studies, in general, corroborate this conclusion. The rhodochrous cultures showed much closer serological relationships with the ' $M$.' rhodochrous and ' $N$.' polychromogenes reference systems than with those provided by the mycobacterial and nocardia cultures. The serological data also support the classification of the rhodochrous cultures into the two large subclusters $\mathrm{I} a$ and $\mathrm{I} b$. The results of the lipid analyses also supported this division, as most of the subcluster i $b$ cultures contained LCN-A type $a$, while those in subclusters I $a$ and I $c$ contained LCN-A type $c$. True mycolic acids are characteristic of mycobacteria, whereas the corresponding nocardomycolic acids were found in Nocardia sensu stricto and the rhodochrous taxon. The serological and lipid studies strengthen the conclusions drawn from the numerical taxonomic studies.

The main aim of this study was to determine the taxonomic relationships between the 'rhodochrous complex' and the genera Mycobacterium and Nocardia. In accordance with some other recent studies (Goodfellow, 1971; Goodfellow et al. 1972; Ridell, 1974), the rhodochrous taxon can be distinguished from these taxa, and also from the proposed genus 
Actinomadura (comprising $N$. dassonvillei, $N$. madurae and $N$. pelletieri). There is, however, no generally accepted taxonomic niche for the rhodochrous taxon: Nocardia (Lechevalier et al. 1971), Gordona (Tsukamura, 1971) and Proactinomyces (Bradley \& Bond, 1974) have each been proposed. We believe, however, that the question of a generic location cannot be settled until the relationships of the rhodochrous taxon to the established genera Arthrobacter and Corynebacterium as well as to Brevibacterium - a 'genus incertae sedis' - have been ascertained. Similarly, additional studies are required before the taxonomic status of the rhodochrous subclusters can be resolved.

We are especially indebted to Mr M. J. Sackin, MRC Microbial Systematics Unit, Leicester University who performed the computer analyses. We are also very grateful to Dr E. F. Lessel of ATCC for preparation and distribution of lyophilized strains, and to the colleagues who kindly provided the cultures. The work carried out at the Institute of Medical Microbiology, University of Göteborg, received financial support from the World Health Organisation, the Swedish National Association against Heart and Chest Diseases, and the Ellen, Walter and Lennart Hesselman's Foundation.

\section{REFERENCES}

BradLey, S. G. (197I). Criteria for definition of Mycobacterium, Nocardia and the rhodochrous complex. Advancing Frontiers of Plant Science 28, 349-362.

Bradley, S. G. \& Bond, J. S. (1974). Taxonomic criteria for mycobacteria and nocardiae. Advances in Applied Microbiology 18, 13I-190.

Cross, T. \& Goodfellow, M. (1973). Taxonomy and classification of the actinomycetes. In Actinomycetales: Characteristics and Practical Importance, pp. I I-I 12. Edited by G. Sykes and F. A. Skinner. London: Academic Press.

Goodfellow, M. (197I). Numerical taxonomy of some nocardioform bacteria. Journal of General Microbiology 69, 33-80.

Goodfellow, M., Fleming, A. \& SACKIN, M. J. (1972). Numerical classification of 'Mycobacterium' rhodochrous and Runyon's group IV mycobacteria. International Journal of Systematic Bacteriology 22, $8 \mathrm{I}-98$.

Goodfellow, M., Minnikin, D. E., Patel, P. V. \& Mordarska, H. (1973). Free nocardomycolic acids in the classification of nocardias and strains of the 'rhodochrous' complex. Journal of General Microbiology 74, $185-188$.

Gordon, R. E. (1966). Some strains in search of a genus - Corynebacterium, Mycobacterium, Nocardia or what? Journal of General Microbiology 43, 329-343.

Gordon, R. E. \& Mirm, J. M. (1957). A comparative study of some strains received as nocardiae. Journal of Bacteriology 73, $15-27$.

Gordon, R. E. \& MinM, J. M. (1959). A comparison of four species of mycobacteria. Journal of General Microbiology 21, 736-748.

KubiCA, G. P. \& ELEVEN Co-workeRs (1972). A co-operative numerical analysis of rapidly growing mycobacteria. Journal of General Microbiology 73, 55-70.

Lechevalier, M. P., Horan, A. C. \& Lechevalier, H. A. (I97I). Lipid composition in the classification of nocardiae and mycobacteria. Journal of Bacteriology 105, 313-318.

LIND, A. (I96I). Serological studies of mycobacteria by means of diffusion-in-gel techniques. IV. The precipitinogenic relationships between different species of mycobacteria with special reference to M. tuberculosis, M. phlei, M. smegmatis and M. avium. International Archives of Allergy 17, 300-322.

MaGnUsson, M. (1962). Specificity of sensitins. III. Further studies in guinea pigs with sensitins of various species of Mycobacterium and Nocardia. American Review of Respiratory Disease 86, 395-404.

MEISSNER, G. \& TWENTY-ONE Co-WORKERS (1974). A co-operative numerical analysis of nonscoto- and nonphoto-chromogenic slowly growing mycobacteria. Journal of General Microbiology 83, 207-235.

MoRdARSKA, H., MoRdARSKI, M. \& Goodfellow, M. (1972). Chemotaxonomic characters and classification of some nocardioform bacteria. Journal of General Microbiology 71, 77-86. 
Norlin, M., Lind, A. \& Ouchterlony, Ö. (1969). A serologically based taxonomic study of Mycobacterium gastri. Zeitschrift fur Immunitätsforschung, Allergie und klinische Immunologie 137, 24I-248.

RIDELL, M. (1974). Serological study of nocardiae and mycobacteria by using 'Mycobacterium' pellegrino and Nocardia corallina precipitation reference systems. International Journal of Systematic Bacteriology 24, 64-72.

Sokal, R. R. \& SNeath, P. H. A. (1963). Principles of Numerical Taxonomy. San Francisco: W. H. Freeman. Tacquet, A., Plancot, M. T., Debruyne, J., Devulder, B., Joseph, M. \& Losfeld, J. (197I). Etudes préliminaries sur la classification numérique des mycobactéries et des nocardias. I. Relations taxonomiques entre Mycobacterium rhodochrous, Mycobacterium pellegrino et les genres Mycobacterium et Nocardia. Annales de l'Institut Pasteur de Lille 22, I2I-I35.

Tsukamura, M. (1971). Proposal of a new genus, Gordona, for slightly acid-fast organisms occurring in sputa of patients with pulmonary disease and in soil. Journal of General Microbiology 68, 15-26.

Tsukamura, M. (1973). A taxonomic study of strains received as 'Mycobacterium' rhodochrous. Japanese Journal of Microbiology 17, 189-197.

WAYNE, L. G. \& FOURTEEN CO-WORKERS (197I). A co-operative numerical analysis of scotochromogenic slowly growing mycobacteria. Journal of General Microbiology 66, 255-27I. 